\title{
The High Frequency Decay Parameter $\kappa$ (Kappa) in the Region of North East India
}

\author{
Renu Yadav1, Dinesh Kumar¹, Sumer Chopra ${ }^{2}$ \\ ${ }^{1}$ Department of Geophysics, Kurukshetra University, Kurukshetra, Haryana, India \\ ${ }^{2}$ Institute of Seismological Research, Gandhinagar, Gujarat, India \\ Email:renuyadav_geokuk@yahoo.com,dineshk5@rediffmail.com, sumer.chopra@gmail.com
}

How to cite this paper: Yadav, R., Kumar, D. and Chopra, S. (2018) The High Frequency Decay Parameter $\kappa$ (Kappa) in the Region of North East India. Open Journal of Earthquake Research, 7, 141-159. https://doi.org/10.4236/ojer.2018.72009

Received: April 26, 2018

Accepted: May 28, 2018

Published: May 31, 2018

Copyright $\odot 2018$ by authors and Scientific Research Publishing Inc. This work is licensed under the Creative Commons Attribution International License (CC BY 4.0).

http://creativecommons.org/licenses/by/4.0/

\section{(c) (i) Open Access}

\begin{abstract}
The high frequency decay parameter $\kappa$ has been considered as one of the important parameters required in the simulation of earthquake strong ground motions necessary for the proper evaluation of seismic hazard of a region. The present study estimated " $k$ " for the highly seismic active region of North East India. The spectral analysis of 598 accelerograms of 32 earthquakes has been done using [1] approach for this purpose. The average values of " $k$ " have been found to be $0.049,0.047$ and 0.040 for L-, T- and V-component respectively. The distance dependence of $\kappa$ is not significant in the region. The $\kappa_{0}(\kappa$ at $\mathrm{R}=$ 0 ) for soft rock stations is found to be more than those of hard rock sites in consistent with other similar studies. The correlation between " $k$ " and earthquake magnitude at most of the stations for the region under study is not significant which indicates that $\kappa$ depends on the site conditions in the region. The $\kappa$ values estimated in the present study are useful for the evaluation of seismic hazard of the region.
\end{abstract}

\section{Keywords}

Kappa, GMPE, Strong Ground Motion, Simulation, Seismic Hazard

\section{Introduction}

The spectral shape of earthquake strong ground motions plays an important role in the simulation of realistic accelerograms using different techniques. The simulated accelerograms are crucial for the proper evaluation of seismic hazard of a region. Different factors including attenuation, velocity and site conditions etc. control the spectral characteristics of the strong ground motions. It has been suggested that the spectrum of strong ground motion from earthquakes is flat above the corner frequency [2] to the maximum frequency $\left(f_{\max }\right)$ after which the 
spectrum decays fast [3]. This phenomenon of high-frequency band limitation of radiated earthquake energy has been given the name "the crashing spectrum syndrome" by [4] and attributed this primarily to the local site effects. [5] suggested the source (fault nonelasticity) as cause for " $f_{\max }$ " not the site. [6] described a site attenuation parameter " $t$ " in the form of exponential decay term $\mathrm{e}^{-\pi f f^{*}}$ to the spectral attenuation of the waves.

[1] introduced a spectral decay parameter $-\kappa$ (Kappa) to model the high frequency spectral attenuation. They defined the parameter " $K$ " as:

$$
A=A_{0} \mathrm{e}^{-\pi f} ; f>f_{E}
$$

where $A_{0}$ depends on the source, epicentral distance and other factors, $f_{E}$ is the frequency above which the spectral amplitude follows an exponential decay. The studies have been done to attribute the origin of " $k$ " to source, site and/or path attenuation. [7] and [8] have suggested that " $k$ " represents the near surface as well as propagation path attenuation. Some studies suggest that " $k$ " is source related [9] [10] [11] [12]. [13] assumes " $K$ " as a parameter related either to source or site effects. It is considered as site parameter by [14] [15] found that " $k$ " was independent of earthquake size within magnitude range $M<3.5$ for the events occurred in the region of Northeastern Sonora, Mexico. [16] also found no correlation between " $k$ " and magnitude.

In spite of the lack of agreement on the physical origin of " $K$ ", it has been widely used in number of seismological applications like computation of site amplification factors, ground motion prediction equations [17] [18]. It has become a standard parameter to constrain attenuation, peak ground acceleration and spectral shape of stochastically generated accelerograms.

In the present study, the high frequency decay parameter " $k$ " has been estimated at different sites and source-receiver distances for the region of North East India. The possible dependence of " $K$ " on distance for hard rock sites and soft soil sites has been investigated. The dependence of " $K$ " on earthquake size has also been examined.

\section{Study Area and Data Used}

The tectonic map of the NE India region has been shown in Figure 1. The collision of India-Eurasian plate (developed Arakan Yoma belt) and under thrusting of Indian plate below the Myanmar plate gives rise to an intricate tectonic zone in North-East Indian region [19]. This region has experienced damaging earthquakes in the past including great earthquakes (1897 and 1950 Assam earthquakes), 1918 Srimangal earthquake (mb 7.6), 1930 Dhubri earthquake (M 7.1). Geologically, this region is mainly divided into five parts as eastern Himalaya, the Mishmi massif, the Indo Myanmar arc, the Brahmaputra valley, and the Shillong plateau [20]. The presence of Mishmi thrust, Lohit thrust, Po Chu fault, and Tidding suture makes the tectonic of Mishmi region more complex having predominant features like Tsangpo suture, Tuting and Bame faults. The Shillong highland is represented by N-S trending Dhansiri and Kulsi faults, N-E aligned 


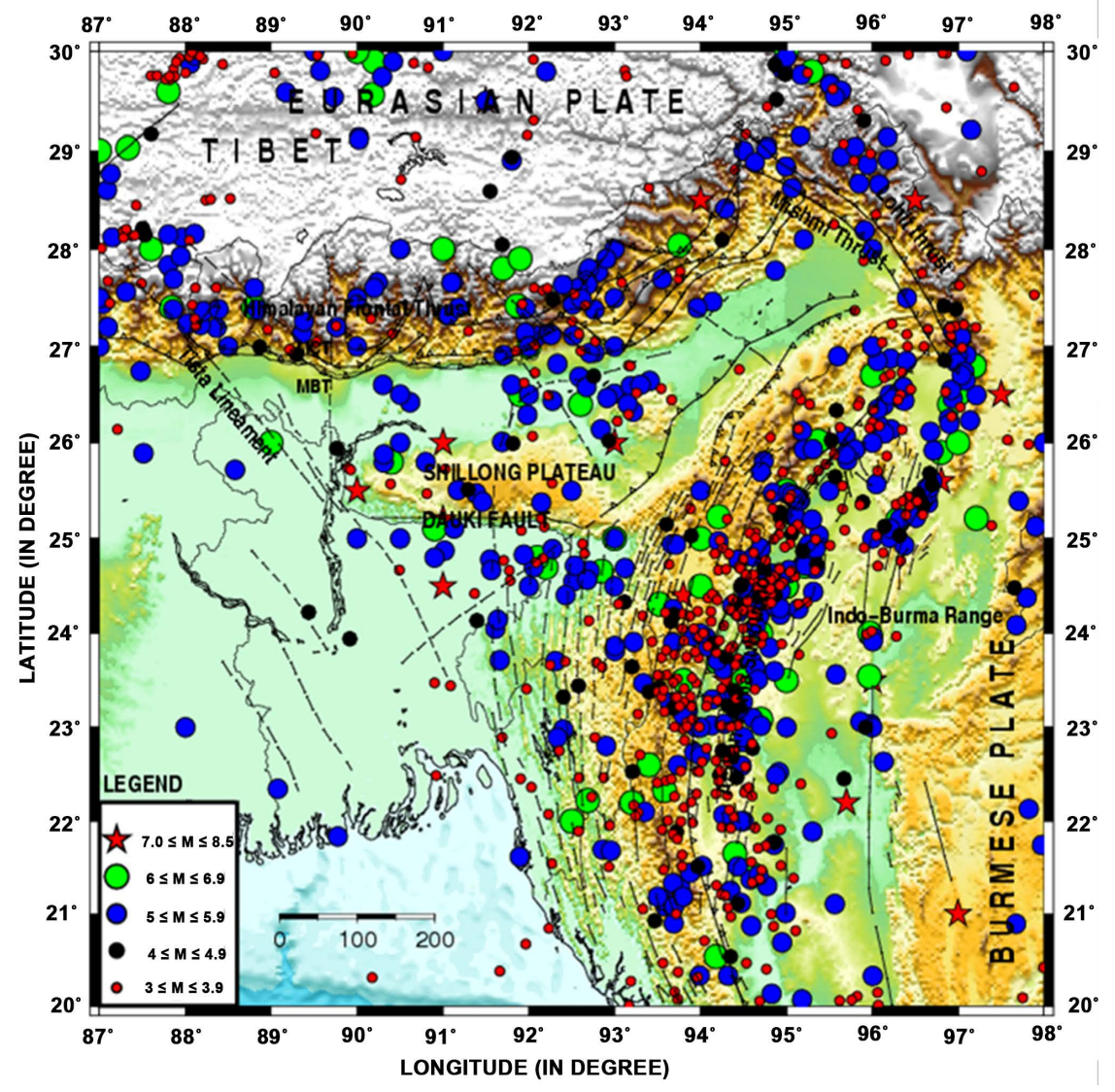

Figure 1. Seismicity along with tectonics of the North-East Region, India.

Barapani Shear region, and Mikir Hills, Dhubri, Sylhet and Duaki faults, and Dudhnai and Kulsi faults. The alignment of Kopili fault in NW-SE direction and in North Dhansiri fault separates Mikir Hills from Shillong highlands [21]. The NE India region is one of the seismically active regions of the world.

A strong motion accelerographs network has been installed in the region by Department of Earthquake Engineering, Indian Institute of Technology, Roorkee with the objectives of studying the strong ground motions characteristics for earthquake engineering purposes. The 598 accelerograms of 32 earthquakes (mb 3.9 - 6.8) recorded at this network has been used in the present analysis. Figure 2 shows the locations of earthquakes and recording stations used in this study. The lists of the earthquakes along with recording stations and geology are given in Table 1.

\section{Methodology}

Different techniques have been reported in the literature to estimate " $k$ " including use of displacement spectra [22], full inversion of source, path and site parameters [23] [24] [25] and broad band inversions [26] [27]. In the present study, the widely used classical method of [1] has been adopted [28] [29] [16]. According to this classical method, Equation (1) can be written as: 


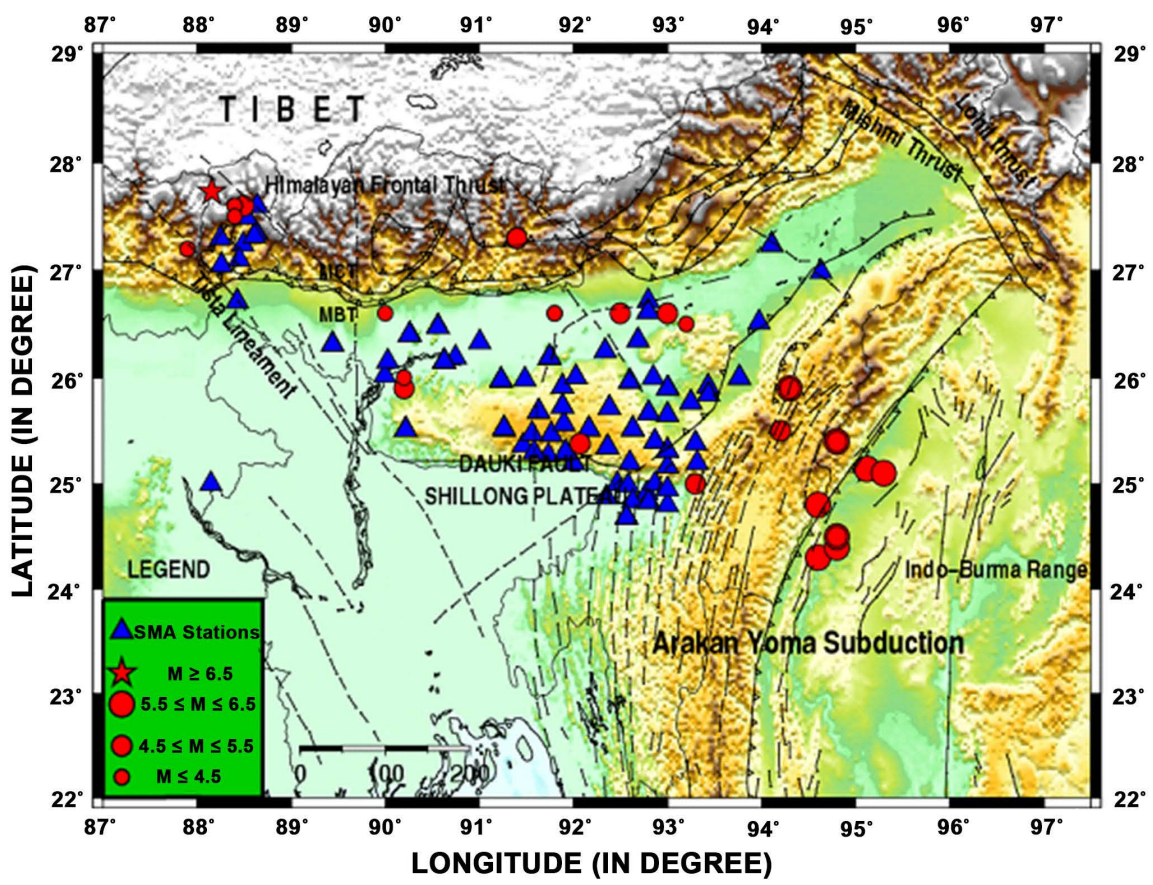

Figure 2. Location of earthquakes and recording stations used in present study.

$$
\ln A=\ln A_{0}-\pi \kappa f
$$

This a linear equation between " $\ln A$ " and frequency " $f$. The " $\kappa$ " can be estimated from the slope $(\mathrm{m})$ of the line (Equation (2)) as:

$$
\kappa=-m / k
$$

The following procedure has been adopted for the estimation of " $\kappa$ ":

1) First the $S$-wave portion of the accelerogram is selected.

2) Fourier transform of the selected wave has been obtained using FFT and plotted the same on log-linear scale i.e. with a logarithmic y-axis (amplitude) and a linear $\mathrm{x}$-axis (frequency).

3) Two frequencies have been selected by visual inspection of the spectrum of S-waves: first at the start of linear downward trend in the spectrum (f1) and second at the end of linear downward trend (f2). The visual inspection of S-wave spectrum in selecting the two frequencies is preferred over the automatic procedure as $\mathrm{f} 1$ and $\mathrm{f} 2$ vary from record to record. The visual inspection avoids the biased estimates of " $\kappa$ ". This procedure has been used in previous studies also (e.g. [28]).

4) A line has fitted between $\mathrm{f} 1$ and $\mathrm{f} 2$ in a least square sense on log-linear plot. The slope (m) of the line gives the value of " $\kappa$ " (Equation (3)).

\section{Results and Discussion}

The values of " $\kappa$ " have been estimated for the three components (N-S, E-W and $\mathrm{Z}$ ) of the recorded accelerograms using the procedure described above. Figure 3 shows the log-linear plots along with the best fitted lines for some of the records. 
Table 1. List of Earthquakes along with recording stations used to compute $\kappa$ and Ns is number of stations.

\begin{tabular}{|c|c|c|c|c|c|c|c|c|c|}
\hline Sr. no & YYYY & MM & $\mathrm{DD}$ & LAT.(N) & LON.(E) & $\mathrm{M}_{\mathrm{W}}$ & $\operatorname{Depth}(\mathrm{Km})$ & Stations & Ns \\
\hline 1 & 1986 & 09 & 10 & 25.38 & 92.07 & 5.2 & 43 & $\begin{array}{l}\text { BAIT, DAUK, KHLI, NONK, } \\
\text { NONP, NONS, PANI, PYNU, } \\
\text { SAIT, UMMU, UMRO, UMSN }\end{array}$ & 12 \\
\hline 2 & 1987 & 05 & 18 & 25.27 & 94.20 & 5.7 & 49 & $\begin{array}{l}\text { BAIT, BAMU, BERL, BOKA, } \\
\text { DIPU, GUNJ, HAFL, HAJA, } \\
\text { HATI, LAIS, NONP, PANI, } \\
\text { SAIT, UMRO }\end{array}$ & 14 \\
\hline 3 & 1988 & 02 & 06 & 24.64 & 91.51 & 5.8 & 33 & $\begin{array}{l}\text { BAIG, BAIT, BAMU, DAUK, } \\
\text { GUNJ, HAFL, HATI, KATA, } \\
\text { KHLI, MAWP, NONK, NONP, } \\
\text { PYNU, SAIT, SHIL, UNMU, } \\
\text { UMRO, UMSN }\end{array}$ & 18 \\
\hline 4 & 1988 & 08 & 06 & 25.14 & 95.12 & 6.8 & 91 & $\begin{array}{c}\text { BAIG, BAIT, BAMU, BERL, } \\
\text { BOKA, CHER, DAUK, DIPU, } \\
\text { DOLO, GUNJ, HAJA, HARE, } \\
\text { HOJA, JELA, JHIR, KALA, } \\
\text { KATA, KHLI, KOOM, LOHA, } \\
\text { MAWK, MAWP, MAWS, } \\
\text { NONK, SILC, UMMU, UMRO, UMSN }\end{array}$ & 28 \\
\hline 5 & 2008 & 03 & 13 & 26.6 & 91.8 & 4.0 & 33 & MNGB, MORB & 2 \\
\hline 6 & 2008 & 05 & 29 & 26.6 & 91.8 & 4.2 & 33 & MNGB, MORB & 2 \\
\hline 7 & 2008 & 12 & 25 & 27.2 & 87.9 & 4.4 & 33 & GANG & 1 \\
\hline 8 & 2009 & 02 & 15 & 26.0 & 90.2 & 4.4 & 39.3 & $\begin{array}{l}\text { BARP, BONG, DHBR, } \\
\text { KOKJ, TURA }\end{array}$ & 5 \\
\hline 9 & 2009 & 02 & 24 & 25.9 & 94.3 & 4.8 & 10 & DIPU, GOLA, LKHM & 3 \\
\hline 10 & 2009 & 08 & 11 & 24.4 & 94.8 & 5.6 & 22 & $\begin{array}{c}\text { BOKO, BONG, DIPU, } \\
\text { GOLP, GWAT, HALK, KARM, KOKJ, } \\
\text { NONG, SILC, TEJP, TURA }\end{array}$ & 12 \\
\hline 11 & 2009 & 08 & 19 & 26.6 & 92.5 & 4.9 & 20 & BOKO, GWAT & 2 \\
\hline 12 & 2009 & 08 & 30 & 25.4 & 94.8 & 5.3 & 85 & $\begin{array}{l}\text { BOKO, DIPU, GWAT, } \\
\text { LKHP, TURA }\end{array}$ & 5 \\
\hline 13 & 2009 & 09 & 03 & 24.3 & 94.6 & 5.9 & 100 & $\begin{array}{c}\text { BOKO, BONG, DIPU, } \\
\text { GOLP, GWAT, HKDI, NONS, SILC, TURA }\end{array}$ & 9 \\
\hline 14 & 2009 & 09 & 21 & 27.3 & 91.5 & 6.2 & 8.0 & $\begin{array}{c}\text { BOKO, BONG, COVB, DRJL, DIPU, GANG, } \\
\text { GOLP, GWAT, KOKJ, NAUG, NONS, } \\
\text { LKHP, TEJP }\end{array}$ & 13 \\
\hline 15 & 2009 & 10 & 29 & 26.6 & 90.0 & 4.2 & 90 & BONG, GOLP, KOKJ, NONS, TURA & 5 \\
\hline 16 & 2009 & 10 & 29 & 27.3 & 91.4 & 5.2 & 5.0 & BONG, GOLP, KOKJ, NONG, TURA & 5 \\
\hline 17 & 2009 & 12 & 29 & 24.5 & 94.8 & 5.5 & 80 & BONG, GOLP, GWAT, HKDI , TEJP, TURA & 6 \\
\hline 18 & 2009 & 12 & 31 & 27.3 & 91.4 & 5.5 & 7.0 & BONG, GOLP, GWAT, KOKJ, TURA & 5 \\
\hline 19 & 2010 & 02 & 26 & 28.5 & 86.7 & 5.4 & 28 & $\begin{array}{l}\text { BONG, DRJL, GANG, GOLP, } \\
\text { GWAT, KOKJ, SILI }\end{array}$ & 7 \\
\hline 20 & 2010 & 03 & 12 & 23.0 & 96.0 & 5.6 & 96 & BONG, GOLP, GWAT, HALK, TURA & 5 \\
\hline 21 & 2010 & 09 & 11 & 25.9 & 90.2 & 5.0 & 20 & BONG, GWAT, KOKJ & 3 \\
\hline 22 & 2010 & 12 & 12 & 25.0 & 93.3 & 4.8 & 15 & KRMJ, NAUG & 2 \\
\hline
\end{tabular}


Continued

\begin{tabular}{|c|c|c|c|c|c|c|c|c|c|}
\hline 23 & 2011 & 02 & 04 & 24.8 & 94.6 & 6.4 & 30 & $\begin{array}{c}\text { COVB, GWAT, JORH, JOWI, KOKJ, } \\
\text { NAUG, SIBS }\end{array}$ & 7 \\
\hline 24 & 2011 & 09 & 18 & 27.6 & 88.2 & 6.8 & 10 & $\begin{array}{c}\text { CHAN, CHPW, COVB, GANG, KRMJ, } \\
\text { KOKJ, MALD, NAUG, PATI, RAXL, SIBS, } \\
\text { SILI, UDHM }\end{array}$ & 13 \\
\hline 25 & 2011 & 09 & 18 & 27.6 & 88.5 & 5.0 & 16 & COVB, GANG & 2 \\
\hline 26 & 2011 & 09 & 18 & 27.5 & 88.4 & 4.5 & 9 & GANG & 1 \\
\hline 27 & 2011 & 09 & 18 & 27.6 & 88.4 & 4.2 & 28 & GANG & 1 \\
\hline 28 & 2011 & 09 & 22 & 27.6 & 88.4 & 3.9 & 30 & GANG & 1 \\
\hline 29 & 2011 & 11 & 21 & 25.1 & 95.3 & 5.8 & 80 & GWAT & 1 \\
\hline 30 & 2012 & 05 & 11 & 26.6 & 93.0 & 5.4 & 20.0 & GOLA, JORH, KOKJ & 3 \\
\hline 31 & 2012 & 07 & 10 & 26.5 & 93.2 & 4.5 & 56 & NAUG & 1 \\
\hline 32 & 2012 & 07 & 14 & 25.5 & 94.2 & 5.5 & 35 & GOLA, JORA, NAUG & 3 \\
\hline
\end{tabular}
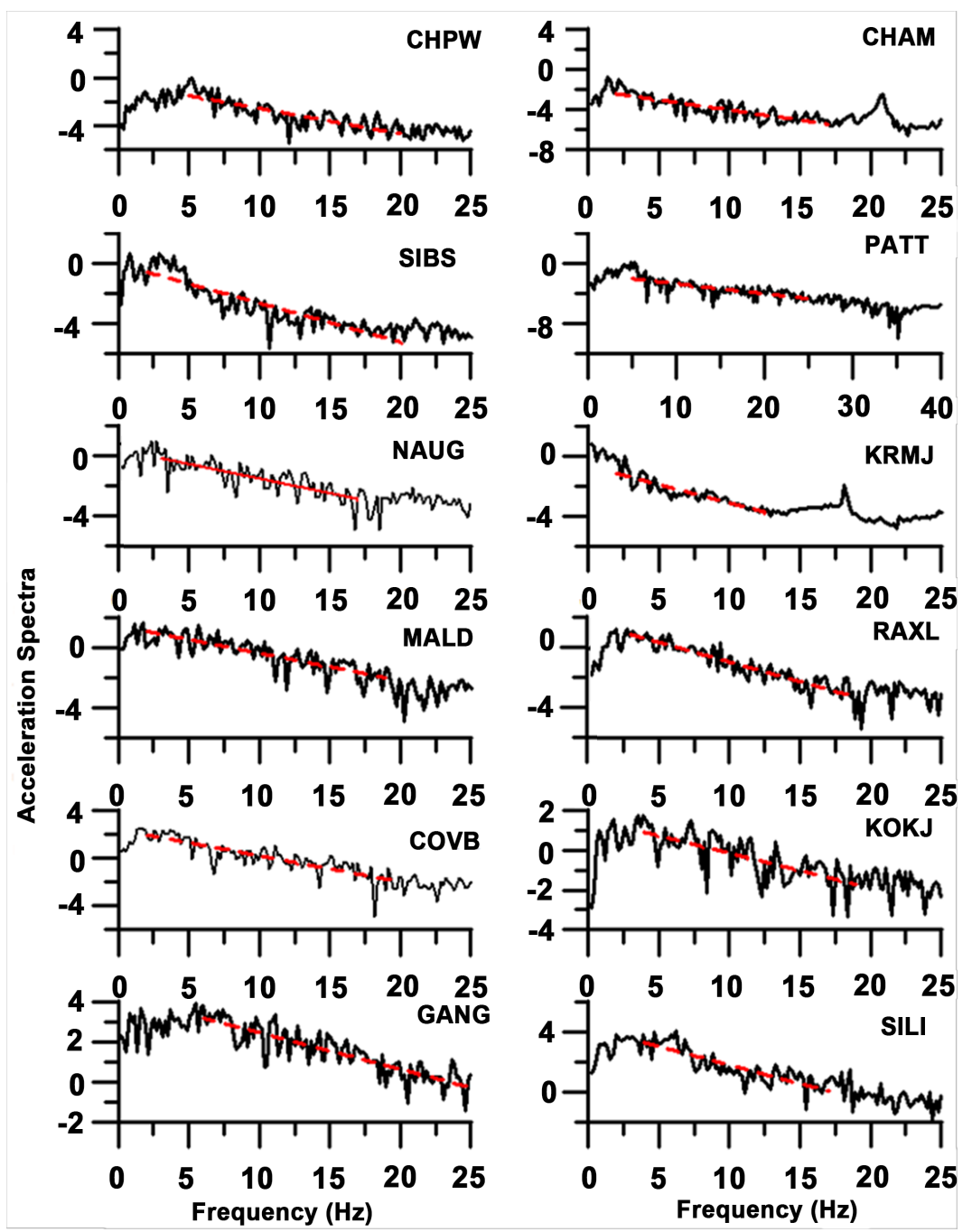

Figure 3. The log-linear plots for estimating " $K$ " along with the best fitted lines for some of the records. 
It has been found that $\mathrm{f} 1$ lies in the range $2-13 \mathrm{~Hz}$ while $\mathrm{f} 2$ is in the range $20-28$ $\mathrm{Hz}$. The estimated values of " $k$ " corresponding to three components for the earthquakes and recording stations are given in Table 2 along with the site geology. The standard deviations are also given in the table. The average values of " $k$ " has been found to be 0.049 (L-component), 0.047 (T-component) and 0.040 (V-component). A comparison between the $\kappa$ values obtained from horizontal and vertical components is shown in Figure 4. The values are found to be similar for most of the events. The vertical estimates are smaller than those of horizontal estimates. This has been observed in other studies [28] [30]. The values obtained in the present study have been compared with those of other regions of the world in Table 3. The estimates are found to be consistent.

The distance dependence of $\kappa$ has been analyzed using the following linear model [1]:

$$
\kappa=\kappa_{0}+\kappa_{R}
$$

where $\kappa_{0}$ is the value of $\kappa$ at distance $R=0$. This model has been used in many studies due to its simplicity of formulation (e.g. [1]; [28]; [31]). $\kappa_{0}$ is believed to be station-dependent and may be related to the near surface attenuation. The distance dependence of $\kappa$ estimated from horizontal and vertical components is shown in Figure 5(a) and Figure 5 (b). The fitted linear model gives the regression.

$$
\kappa=0.037+0.0000158 R \quad \text { For vertical component }
$$

and

$$
\kappa=0.041+0.0000326 R \text { For horizontal component }
$$

We note that distance dependence for both the components is not significant. The values of $\kappa_{0}$ as 0.041 (horizontal component) and 0.037 (vertical component) represent the overall value for the region. The difference in these two values indicates that site response is different for different components as has been observed in site amplification studies. The estimate of $\kappa_{0}$ (vertical) is useful along with $\mathrm{H} / \mathrm{V}$ ratio for the first order estimation of site effect where site-specific borehole data is not available as suggested by [30].

Figure 6(a) and Figure 6(b) show the distance dependence of $\kappa$ on hard rock sites and soft soil sites separately. The linear fit gives the following relations:

$$
\kappa=0.034+0.0000158 R \text { For hard rock sites }
$$

and

$$
\kappa=0.037+0.000024 R \text { For soft rock sites }
$$

The $\kappa_{0}$ for soft rock stations is found to be more than those of hard rock sites. The ratio of $\kappa_{0}$ for soft rock to hard rock is equal to 1.09. Similar observations have been found to be in different studies for other regions. [28] found that kappa depends on both local geology (Soil or Rock) and source to site distance in France. They have estimated $\kappa_{0}$ (soil) $=0.0270$ and $\kappa_{0}$ (rock) $=0.0207$. [32] has found $\kappa_{0}$ for soil as 0.036 and $\kappa_{0}$ for rock as 0.030 for Southern California. 
Table 2. The estimated values of $\kappa$ for the three components of earthquakes along with recording stations with site geology.

\begin{tabular}{|c|c|c|c|c|c|c|c|c|c|}
\hline Sr. no. & $\begin{array}{l}\text { Date of } \\
\text { Event }\end{array}$ & Station & Kappa-L & SD & Kappa-T & SD & Kappa-V & SD & Geology \\
\hline \multirow[t]{12}{*}{1} & $1986 / 09 / 10$ & BAIT & 0.031 & 0.0051 & 0.011 & 0.0049 & 0.015 & 0.0039 & Soft soil \\
\hline & & DAUK & 0.053 & 0.0048 & 0.050 & 0.0048 & 0.037 & 0.0044 & Soft soil \\
\hline & & KHLI & 0.035 & 0.0046 & 0.033 & 0.0056 & 0.037 & 0.0052 & Hard rock \\
\hline & & NONK & 0.021 & 0.0050 & 0.028 & 0.0055 & 0.020 & 0.0036 & Hard rock \\
\hline & & NONP & 0.021 & 0.0032 & 0.014 & 0.0031 & 0.051 & 0.0057 & Hard rock \\
\hline & & NONS & 0.023 & 0.0033 & 0.029 & 0.0048 & 0.033 & 0.0040 & Hard rock \\
\hline & & PANI & 0.031 & 0.0032 & 0.018 & 0.0033 & 0.039 & 0.0062 & Hard rock \\
\hline & & PYNU & 0.036 & 0.0031 & 0.023 & 0.0045 & 0.019 & 0.0056 & Hard rock \\
\hline & & SAIT & 0.024 & 0.0037 & 0.026 & 0.0041 & 0.027 & 0.0041 & Soft soil \\
\hline & & UMMU & 0.035 & 0.0032 & 0.024 & 0.0041 & 0.043 & 0.0061 & Hard rock \\
\hline & & UMRO & 0.036 & 0.0055 & 0.027 & 0.0047 & 0.027 & 0.0056 & Soft soil \\
\hline & & UMSN & 0.013 & 0.0051 & 0.014 & 0.0044 & 0.030 & 0.0038 & Hard rock \\
\hline \multirow[t]{14}{*}{2} & $1987 / 05 / 18$ & BAIT & 0.027 & 0.0032 & 0.023 & 0.0035 & 0.020 & 0.0049 & Soft soil \\
\hline & & BAMU & 0.024 & 0.0052 & 0.028 & 0.0062 & 0.034 & 0.0056 & Soft soil \\
\hline & & BERL & 0.036 & 0.0061 & 0.029 & 0.0057 & 0.024 & 0.0052 & Soft soil \\
\hline & & BOKA & 0.019 & 0.0040 & 0.034 & 0.0046 & 0.017 & 0.0048 & Soft soil \\
\hline & & DIPU & 0.042 & 0.0072 & 0.039 & 0.0070 & 0.028 & 0.0049 & Soft soil \\
\hline & & GUNJ & 0.031 & 0.0050 & 0.038 & 0.0048 & 0.027 & 0.0073 & Soft soil \\
\hline & & HAFL & 0.030 & 0.0039 & 0.029 & 0.0035 & 0.039 & 0.0073 & Soft soil \\
\hline & & HAJA & 0.026 & 0.0055 & 0.028 & 0.0054 & 0.029 & 0.0063 & Soft soil \\
\hline & & HATI & 0.032 & 0.0048 & 0.022 & 0.0045 & 0.027 & 0.0039 & Soft soil \\
\hline & & LAIS & 0.15 & 0.005 & 0.036 & 0.0037 & 0.043 & 0.0046 & Hard rock \\
\hline & & NONP & 0.022 & 0.0042 & 0.023 & 0.0040 & 0.041 & 0.0068 & Hard rock \\
\hline & & PANI & 0.032 & 0.0066 & 0.033 & 0.0068 & 0.030 & 0.0092 & Hard rock \\
\hline & & SAIT & 0.030 & 0.0075 & 0.022 & 0.0072 & 0.032 & 0.0119 & Soft soil \\
\hline & & UMRO & 0.053 & 0.0074 & 0.034 & 0.0063 & 0.033 & 0.0084 & Soft soil \\
\hline \multirow[t]{12}{*}{3} & $1988 / 02 / 06$ & BAIG & 0.043 & 0.0049 & 0.041 & 0.0047 & 0.031 & 0.0072 & Soft soil \\
\hline & & BAIT & 0.052 & 0.0051 & 0.038 & 0.0047 & - & - & Soft soil \\
\hline & & BAMU & 0.028 & 0.0043 & 0.027 & 0.0048 & 0.051 & 0.0071 & Soft soil \\
\hline & & DAUK & 0.034 & 0.0040 & 0.033 & 0.0039 & 0.023 & 0.0080 & Soft soil \\
\hline & & GUNJ & 0.035 & 0.0037 & 0.033 & 0.0037 & 0.034 & 0.0052 & Soft soil \\
\hline & & HAFL & 0.026 & 0.0033 & 0.033 & 0.0035 & 0.043 & 0.0086 & Soft soil \\
\hline & & HATI & 0.034 & 0.0051 & 0.034 & 0.0045 & 0.036 & 0.0090 & Soft soil \\
\hline & & KATA & 0.012 & 0.0035 & 0.028 & 0.0031 & 0.035 & 0.0033 & Soft soil \\
\hline & & KHLI & 0.033 & 0.0059 & 0.034 & 0.0048 & 0.053 & 0.0084 & Hard rock \\
\hline & & MAWP & 0.019 & 0.0051 & 0.016 & 0.0048 & 0.032 & 0.0104 & Hard rock \\
\hline & & NONK & 0.040 & 0.0045 & 0.036 & 0.0038 & 0.015 & 0.0039 & Hard rock \\
\hline & & NONP & 0.034 & 0.0070 & 0.026 & 0.0064 & 0.050 & 0.0059 & Hard rock \\
\hline
\end{tabular}


R. Yadav et al.

\section{Continued}

\begin{tabular}{|c|c|c|c|c|c|c|c|c|c|}
\hline & & PYNU & 0.041 & 0.0047 & 0.040 & 0.0059 & 0.014 & 0.0052 & Hard rock \\
\hline & & SAIT & 0.027 & 0.0038 & 0.023 & 0.0055 & 0.015 & 0.0049 & Soft soil \\
\hline & & SHIL & 0.033 & 0.0050 & 0.029 & 0.0046 & 0.024 & 0.0053 & Hard rock \\
\hline & & UMMU & 0.045 & 0.0109 & 0.060 & 0.0134 & 0.049 & 0.0124 & Hard rock \\
\hline & & UMRO & 0.037 & 0.0048 & 0.033 & 0.0035 & 0.041 & 0.0050 & Soft soil \\
\hline & & UMSN & 0.017 & 0.0040 & 0.019 & 0.0033 & 0.024 & 0.0043 & Hard rock \\
\hline \multirow[t]{33}{*}{4} & $1988 / 08 / 06$ & BAIG & 0.056 & 0.0053 & 0.018 & 0.0051 & - & - & Soft soil \\
\hline & & BAIT & 0.019 & 0.0040 & 0.019 & 0.0035 & 0.012 & 0.0126 & Soft soil \\
\hline & & BAMU & 0.026 & 0.0042 & 0.022 & 0.0036 & 0.033 & 0.0045 & Soft soil \\
\hline & & BERL & 0.034 & 0.0040 & 0.021 & 0.0032 & 0.021 & 0.0035 & Soft soil \\
\hline & & BOKA & 0.017 & 0.0039 & 0.007 & 0.0039 & 0.005 & 0.0035 & Soft soil \\
\hline & & CHER & 0.034 & 0.0037 & 0.032 & 0.0040 & 0.013 & 0.0034 & Hard rock \\
\hline & & DAUK & 0.036 & 0.0030 & 0.040 & 0.0034 & 0.021 & 0.0041 & Soft soil \\
\hline & & DIPU & 0.026 & 0.0039 & 0.029 & 0.0036 & 0.047 & 0.0116 & Soft soil \\
\hline & & DOLO & 0.036 & 0.0037 & 0.042 & 0.0036 & 0.016 & 0.0043 & Soft soil \\
\hline & & GUNJ & 0.027 & 0.0033 & 0.026 & 0.0042 & 0.027 & 0.0049 & Soft soil \\
\hline & & HAJA & 0.046 & 0.0102 & 0.033 & 0.0083 & 0.027 & 0.0074 & Soft soil \\
\hline & & HARE & 0.031 & 0.0040 & 0.036 & 0.0037 & 0.027 & 0.0039 & Soft soil \\
\hline & & HOJA & 0.021 & 0.0037 & 0.034 & 0.0057 & 0.014 & 0.0067 & Soft soil \\
\hline & & JELA & 0.042 & 0.0047 & 0.045 & 0.0051 & 0.037 & 0.0043 & Soft soil \\
\hline & & JHIR & 0.038 & 0.0047 & 0.042 & 0.0056 & 0.034 & 0.0062 & Soft soil \\
\hline & & KALA & 0.036 & 0.0047 & 0.042 & 0.0056 & 0.034 & 0.0062 & Soft soil \\
\hline & & KATA & 0.074 & 0.0097 & 0.074 & 0.0098 & 0.035 & 0.0050 & Soft soil \\
\hline & & KHLI & 0.024 & 0.0073 & 0.022 & 0.0115 & 0.026 & 0.0045 & Hard rock \\
\hline & & KOOM & 0.036 & 0.0041 & 0.045 & 0.0061 & 0.040 & 0.0057 & Soft soil \\
\hline & & LOHA & 0.041 & 0.0052 & 0.034 & 0.0057 & 0.029 & 0.0043 & Soft soil \\
\hline & & MAWK & 0.040 & 0.0082 & 0.029 & 0.0073 & 0.007 & 0.0105 & Hard rock \\
\hline & & MAWP & 0.026 & 0.0040 & 0.038 & 0.0037 & 0.018 & 0.0048 & Hard rock \\
\hline & & MAWS & 0.016 & 0.0031 & 0.015 & 0.0041 & 0.025 & 0.0062 & Hard rock \\
\hline & & NONK & 0.026 & 0.0032 & 0.030 & 0.0029 & 0.013 & 0.0056 & Hard rock \\
\hline & & NONS & 0.030 & 0.0081 & 0.042 & 0.0064 & 0.054 & 0.0071 & Hard rock \\
\hline & & PANI & 0.045 & 0.0087 & 0.020 & 0.0096 & 0.021 & 0.0048 & Hard rock \\
\hline & & PYNU & 0.035 & 0.0068 & 0.062 & 0.0082 & 0.019 & 0.0094 & Hard rock \\
\hline & & SAIT & 0.025 & 0.0048 & 0.022 & 0.0069 & 0.020 & 0.0108 & Soft soil \\
\hline & & SHIL & 0.028 & 0.0071 & 0.013 & 0.0082 & 0.046 & 0.0115 & Hard rock \\
\hline & & SILC & 0.039 & 0.0061 & 0.038 & 0.0054 & 0.035 & 0.0061 & Soft soil \\
\hline & & UMMU & 0.041 & 0.0084 & 0.044 & 0.0104 & 0.022 & 0.0143 & Hard rock \\
\hline & & UMRO & 0.040 & 0.0061 & 0.039 & 0.0052 & 0.029 & 0.0043 & Soft soil \\
\hline & & UMSN & 0.026 & 0.0077 & 0.011 & 0.0076 & 0.022 & 0.0089 & Hard rock \\
\hline \multirow[t]{2}{*}{5} & $2008 / 03 / 13$ & MNG & 0.044 & 0.0047 & 0.038 & 0.0038 & 0.024 & 0.0042 & Soft soil \\
\hline & & MOR & 0.036 & 0.0037 & 0.028 & 0.0042 & 0.017 & 0.0056 & Soft soil \\
\hline
\end{tabular}




\section{Continued}

\begin{tabular}{|c|c|c|c|c|c|c|c|c|c|}
\hline \multirow[t]{2}{*}{6} & $2008 / 05 / 29$ & MNG & 0.039 & 0.0034 & 0.036 & 0.0052 & 0.030 & 0.0048 & Soft soil \\
\hline & & MOR & 0.024 & 0.0041 & 0.028 & 0.0050 & 0.021 & 0.0053 & Soft soil \\
\hline 7 & $2008 / 12 / 25$ & GANG & 0.062 & 0.0068 & 0.081 & 0.0050 & 0.045 & 0.0073 & Hard rock \\
\hline \multirow[t]{5}{*}{8} & $2009 / 02 / 15$ & BARP & 0.134 & 0.0195 & 0.116 & 0.157 & 0.020 & 0.0217 & Soft soil \\
\hline & & BONG & 0.036 & 0.043 & 0.034 & 0.061 & 0.049 & 0.0071 & Soft soil \\
\hline & & DHBR & 0.051 & 0.0050 & 0.054 & 0.0049 & 0.051 & 0.0058 & Soft soil \\
\hline & & KOKJ & 0.055 & 0.0089 & 0.030 & 0.0054 & 0.025 & 0.0129 & Soft soil \\
\hline & & TURA & 0.063 & 0.0067 & 0.067 & 0.0055 & 0.067 & 0.0067 & Soft soil \\
\hline \multirow[t]{3}{*}{9} & $2009 / 02 / 24$ & DIPU & 0.024 & 0.0056 & 0.035 & 0.0058 & 0.025 & 0.0042 & Soft soil \\
\hline & & GOLA & 0.051 & 0.0081 & 0.043 & 0.0075 & 0.030 & 0.0068 & Soft soil \\
\hline & & LKHM & 0.075 & 0.0081 & 0.076 & 0.0069 & 0.045 & 0.0095 & Soft soil \\
\hline \multirow[t]{12}{*}{10} & $2009 / 08 / 11$ & ВОКО & 0.046 & 0.0069 & 0.036 & 0.0069 & 0.017 & 0.0061 & Soft soil \\
\hline & & BONG & 0.061 & 0.0073 & 0.067 & 0.0057 & 0.061 & 0.0065 & Soft soil \\
\hline & & DIPU & 0.054 & 0.0066 & 0.049 & 0.0054 & 0.032 & 0.0064 & Soft soil \\
\hline & & GOLP & 0.070 & 0.0090 & 0.053 & 0.0082 & 0.057 & 0.0059 & Soft soil \\
\hline & & GWAT & 0.037 & 0.0052 & 0.044 & 0.0050 & 0.062 & 0.0059 & Soft soil \\
\hline & & HALK & 0.121 & 0.0097 & 0.136 & 0.0093 & 0.104 & 0.0116 & Soft soil \\
\hline & & KARM & 0.109 & 0.0073 & 0.112 & 0.0087 & 0.062 & 0.0068 & Soft soil \\
\hline & & KOKJ & 0.049 & 0.0052 & 0.042 & 0.0057 & 0.044 & 0.0074 & Soft soil \\
\hline & & NONG & 0.031 & 0.0051 & 0.017 & 0.0053 & 0.017 & 0.0053 & Hard rock \\
\hline & & SILC & 0.105 & 0.0079 & 0.110 & 0.0094 & 0.058 & 0.0046 & Soft soil \\
\hline & & TEJP & 0.030 & 0.0065 & 0.029 & 0.0066 & 0.028 & 0.0076 & Soft soil \\
\hline & & TURA & 0.063 & 0.0069 & 0.063 & 0.0061 & 0.053 & 0.0056 & Soft soil \\
\hline \multirow[t]{2}{*}{11} & 2009/08/19 & BOKO & 0.029 & 0.0037 & 0.049 & 0.0039 & 0.025 & 0.0036 & Soft soil \\
\hline & & GUAT & 0.043 & 0.0031 & 0.041 & 0.0032 & 0.046 & 0.0035 & Soft soil \\
\hline \multirow[t]{5}{*}{12} & $2009 / 08 / 30$ & BOKA & 0.029 & 0.0033 & 0.031 & 0.0034 & 0.020 & 0.0041 & Soft soil \\
\hline & & DIPU & 0.039 & 0.0037 & 0.041 & 0.0032 & 0.027 & 0.0026 & Soft soil \\
\hline & & GUA & 0.049 & 0.0036 & 0.048 & 0.0038 & 0.052 & 0.0040 & Soft soil \\
\hline & & LKH & 0.047 & 0.0037 & 0.054 & 0.0038 & 0.032 & 0.0036 & Soft soil \\
\hline & & TURA & 0.055 & 0.0031 & 0.059 & 0.0034 & 0.051 & 0.0036 & Soft soil \\
\hline \multirow[t]{9}{*}{13} & $2009 / 09 / 03$ & BOKA & 0.026 & 0.0030 & 0.020 & 0.0037 & 0.007 & 0.0042 & Soft soil \\
\hline & & BONG & 0.052 & 0.0037 & 0.048 & 0.0047 & 0.068 & 0.0045 & Soft soil \\
\hline & & DIPU & 0.038 & 0.0042 & 0.034 & 0.0045 & 0.033 & 0.0037 & Soft soil \\
\hline & & GOLP & 0.027 & 0.0032 & 0.039 & 0.0035 & 0.032 & 0.0044 & Soft soil \\
\hline & & GUA & 0.042 & 0.0042 & 0.032 & 0.0040 & 0.036 & 0.0040 & Soft soil \\
\hline & & HKD & 0.090 & 0.0042 & 0.105 & 0.0041 & 0.061 & 0.041 & Soft soil \\
\hline & & NONS & 0.030 & 0.0046 & 0.023 & 0.0044 & 0.046 & 0.0045 & Hard rock \\
\hline & & SILC & 0.079 & 0.0039 & 0.087 & 0.0043 & 0.052 & 0.0054 & Soft soil \\
\hline & & TURA & 0.053 & 0.0043 & 0.039 & 0.0042 & 0.037 & 0.0039 & Soft soil \\
\hline \multirow[t]{2}{*}{14} & $2009 / 09 / 21$ & ВОКО & 0.051 & 0.0047 & 0.054 & 0.0033 & 0.042 & 0.0038 & Soft soil \\
\hline & & BONG & 0.062 & 0.0041 & 0.070 & 0.0040 & 0.057 & 0.0041 & Soft soil \\
\hline
\end{tabular}


R. Yadav et al.

\section{Continued}

\begin{tabular}{|c|c|c|c|c|c|c|c|c|c|}
\hline & & COBV & 0.086 & 0.0037 & 0.075 & 0.0036 & 0.063 & 0.0050 & Soft soil \\
\hline & & DJL & 0.071 & 0.0040 & 0.095 & 0.0050 & 0.062 & 0.0045 & Hard rock \\
\hline & & DIPU & 0.055 & 0.0041 & 0.072 & 0.0039 & 0.056 & 0.0039 & Soft soil \\
\hline & & GANG & 0.067 & 0.0032 & 0.078 & 0.0039 & 0.051 & 0.0039 & Hard rock \\
\hline & & GOLP & 0.068 & 0.0033 & 0.065 & 0.0045 & 0.062 & 0.0040 & Soft soil \\
\hline & & GUAT & 0.051 & 0.0050 & 0.041 & 0.0051 & 0.058 & 0.0043 & Soft soil \\
\hline & & KOKJ & 0.048 & 0.0036 & 0.053 & 0.0046 & 0.063 & 0.0047 & Soft soil \\
\hline & & NAUG & 0.070 & 0.0028 & 0.075 & 0.0030 & 0.069 & 0.0036 & Soft soil \\
\hline & & NONS & 0.057 & 0.0044 & 0.051 & 0.0049 & 0.074 & 0.0062 & Hard rock \\
\hline & & LKH & 0.076 & 0.0050 & 0.095 & 0.0039 & 0.061 & 0.0048 & Soft soil \\
\hline & & TEJP & 0.064 & 0.0037 & 0.065 & 0.0065 & 0.064 & 0.0046 & Soft soil \\
\hline \multirow[t]{5}{*}{15} & $2009 / 10 / 29$ & BONG & 0.066 & 0.0063 & 0.076 & 0.0073 & 0.063 & 0.0048 & Soft soil \\
\hline & & GOLP & 0.092 & 0.0051 & 0.082 & 0.0060 & 0.065 & 0.0047 & Soft soil \\
\hline & & KOKJ & 0.078 & 0.0068 & 0.070 & 0.0069 & 0.037 & 0.0050 & Soft soil \\
\hline & & NONS & 0.078 & 0.0079 & 0.076 & 0.0079 & 0.065 & 0.0069 & Hard rock \\
\hline & & TURA & 0.111 & 0.0096 & 0.118 & 0.0091 & 0.123 & 0.0084 & Soft soil \\
\hline \multirow[t]{5}{*}{16} & $2009 / 10 / 29$ & BONG & 0.050 & 0.0051 & 0.066 & 0.0050 & 0.066 & 0.0044 & Soft soil \\
\hline & & GOLP & 0.029 & 0.0097 & 0.022 & 0.0060 & 0.031 & 0.0103 & Soft soil \\
\hline & & KOKJ & 0.030 & 0.0064 & 0.018 & 0.0072 & 0.035 & 0.0132 & Soft soil \\
\hline & & NONS & 0.068 & 0.0054 & 0.058 & 0.0058 & 0.049 & 0.0049 & Hard rock \\
\hline & & TURA & 0.055 & 0.0063 & 0.052 & 0.0062 & 0.037 & 0.0053 & Soft soil \\
\hline \multirow[t]{6}{*}{17} & $2009 / 12 / 29$ & BONG & 0.061 & 0.0058 & 0.055 & 0.0049 & 0.081 & 0.0076 & Soft soil \\
\hline & & GOLP & 0.066 & 0.0062 & 0.037 & 0.0091 & 0.051 & 0.0054 & Soft soil \\
\hline & & GWAT & 0.051 & 0.0068 & 0.036 & 0.0054 & 0.084 & 0.0067 & Soft soil \\
\hline & & HALK & 0.104 & 0.0053 & 0.101 & 0.0066 & - & - & Soft soil \\
\hline & & TEJP & 0.023 & 0.0055 & 0.028 & 0.0046 & 0.041 & 0.0050 & Soft soil \\
\hline & & TURA & 0.040 & 0.0057 & 0.040 & 0.0075 & 0.038 & 0.0045 & Soft soil \\
\hline \multirow[t]{5}{*}{18} & $2009 / 12 / 31$ & BONG & 0.058 & 0.0056 & 0.063 & 0.0068 & 0.058 & 0.0048 & Soft soil \\
\hline & & GOLP & 0.056 & 0.0054 & 0.043 & 0.0047 & 0.057 & 0.0050 & Soft soil \\
\hline & & GWAT & 0.034 & 0.0061 & 0.035 & 0.0064 & 0.046 & 0.0052 & Soft soil \\
\hline & & KOKJ & 0.061 & 0.0053 & 0.071 & 0.0056 & 0.066 & 0.0059 & Soft soil \\
\hline & & TURA & 0.094 & 0.0050 & 0.091 & 0.0063 & 0.080 & 0.0042 & Soft soil \\
\hline \multirow[t]{7}{*}{19} & $2010 / 02 / 26$ & BONG & 0.082 & 0.0067 & 0.070 & 0.0049 & 0.066 & 0.0051 & Soft soil \\
\hline & & DRJL & 0.062 & 0.0059 & 0.044 & 0.0054 & 0.031 & 0.0061 & Hard rock \\
\hline & & GANG & 0.069 & 0.0047 & 0.057 & 0.0070 & 0.014 & 0.0060 & Hard rock \\
\hline & & GOLP & 0.084 & 0.0047 & 0.087 & 0.0051 & 0.067 & 0.0051 & Soft soil \\
\hline & & GWAT & 0.069 & 0.0068 & 0.070 & 0.0057 & 0.063 & 0.0062 & Soft soil \\
\hline & & KOKJ & 0.063 & 0.0069 & 0.082 & 0.0064 & 0.033 & 0.0056 & Soft soil \\
\hline & & SILI & 0.070 & 0.0053 & 0.072 & 0.0056 & 0.025 & 0.0060 & Soft soil \\
\hline \multirow[t]{2}{*}{20} & $2010 / 03 / 12$ & BONG & 0.041 & 0.0044 & 0.052 & 0.0052 & 0.076 & 0.0059 & Soft soil \\
\hline & & GOLP & 0.069 & 0.0121 & 0.060 & 0.0062 & 0.055 & 0.0084 & Soft soil \\
\hline
\end{tabular}




\section{Continued}

\begin{tabular}{|c|c|c|c|c|c|c|c|c|c|}
\hline & & GWAT & 0.028 & 0.0047 & 0.027 & 0.0071 & 0.061 & 0.0074 & Soft soil \\
\hline & & HALK & 0.095 & 0.0052 & 0.101 & 0.0056 & 0.030 & 0.0052 & Soft soil \\
\hline & & TURA & 0.062 & 0.0054 & 0.064 & 0.0055 & 0.054 & 0.0060 & Soft soil \\
\hline \multirow[t]{3}{*}{21} & $2010 / 09 / 11$ & BONG & 0.032 & 0.0065 & 0.042 & 0.0048 & 0.049 & 0.0050 & Soft soil \\
\hline & & GWAT & 0.033 & 0.0044 & 0.033 & 0.0055 & 0.051 & 0.0046 & Soft soil \\
\hline & & KOKJ & 0.042 & 0.0059 & 0.044 & 0.0049 & 0.073 & 0.0048 & Soft soil \\
\hline \multirow[t]{2}{*}{22} & $2010 / 12 / 12$ & KRMJ & 0.132 & 0.0077 & 0.144 & 0.0099 & 0.091 & 0.0078 & Soft soil \\
\hline & & NAUG & 0.060 & 0.0061 & 0.057 & 0.0058 & 0.049 & 0.0063 & Soft soil \\
\hline \multirow[t]{6}{*}{23} & $2011 / 02 / 04$ & COVB & 0.059 & 0.0091 & 0.047 & 0.0082 & 0.038 & 0.0066 & Soft soil \\
\hline & & GWAT & 0.044 & 0.0040 & 0.034 & 0.0069 & 0.049 & 0.0047 & Soft soil \\
\hline & & JORH & 0.045 & 0.0039 & 0.035 & 0.0046 & 0.016 & 0.0050 & Soft soil \\
\hline & & JOWI & 0.049 & 0.0076 & 0.065 & 0.0073 & 0.011 & 0.0058 & Soft soil \\
\hline & & KOKJ & 0.030 & 0.0049 & - & - & 0.033 & 0.0057 & Soft soil \\
\hline & & NAUG & 0.029 & 0.0047 & 0.034 & 0.0047 & 0.028 & 0.0052 & Soft soil \\
\hline \multirow[t]{13}{*}{24} & $2011 / 09 / 18$ & CHAM & 0.064 & 0.0057 & 0.093 & 0.0067 & 0.040 & 0.0048 & Hard rock \\
\hline & & CHPW & 0.081 & 0.0081 & 0.102 & 0.0093 & 0.019 & 0.0056 & Hard rock \\
\hline & & COVB & 0.069 & 0.0045 & 0.056 & 0.0043 & 0.037 & 0.0063 & Soft soil \\
\hline & & GANG & 0.057 & 0.0056 & 0.050 & 0.0061 & 0.046 & 0.0053 & Hard rock \\
\hline & & KRMJ & 0.078 & 0.0055 & 0.084 & 0.0077 & 0.102 & 0.0084 & Soft soil \\
\hline & & KOKJ & 0.055 & 0.0063 & 0.054 & 0.0062 & 0.049 & 0.0080 & Soft soil \\
\hline & & MALD & 0.058 & 0.0044 & 0.060 & 0.0044 & 0.077 & 0.0068 & Soft soil \\
\hline & & NAUG & 0.062 & 0.0061 & 0.067 & 0.0044 & 0.053 & 0.0052 & Soft soil \\
\hline & & PATI & 0.057 & 0.0086 & 0.081 & 0.0068 & 0.070 & 0.0071 & Hard rock \\
\hline & & RAXL & 0.083 & 0.0039 & 0.061 & 0.0037 & 0.022 & 0.0061 & Soft soil \\
\hline & & SIBS & 0.097 & 0.0077 & 0.098 & 0.0077 & - & - & Soft soil \\
\hline & & SILI & 0.079 & 0.0064 & 0.095 & 0.0074 & 0.032 & 0.0080 & Soft soil \\
\hline & & UDHM & 0.093 & 0.0067 & 0.077 & 0.0072 & 0.047 & 0.0064 & Soft soil \\
\hline \multirow[t]{2}{*}{25} & $2011 / 09 / 18$ & COVB & 0.069 & 0.0066 & 0.097 & 0.0091 & 0.060 & 0.0075 & Soft soil \\
\hline & & GANG & 0.072 & 0.0058 & 0.032 & 0.0082 & 0.032 & 0.0076 & Hard rock \\
\hline 26 & $2011 / 09 / 18$ & GANG & 0.062 & 0.0054 & 0.054 & 0.0048 & 0.062 & 0.0101 & Hard rock \\
\hline 27 & $2011 / 09 / 18$ & GANG & 0.036 & 0.0053 & 0.041 & 0.0047 & 0.019 & 0.0071 & Hard rock \\
\hline 28 & $2011 / 09 / 22$ & GANG & 0.039 & 0.0051 & 0.028 & 0.0055 & 0.028 & 0.0062 & Hard rock \\
\hline 29 & $2011 / 11 / 21$ & GWAT & 0.035 & 0.0098 & 0.035 & 0.0098 & 0.017 & 0.0078 & Soft soil \\
\hline \multirow[t]{3}{*}{30} & $2012 / 05 / 11$ & GOLA & 0.072 & 0.0186 & 0.026 & 0.0124 & 0.049 & 0.0153 & Soft soil \\
\hline & & JORH & 0.039 & 0.0085 & 0.040 & 0.0065 & 0.023 & 0.0070 & Soft soil \\
\hline & & KOKJ & 0.049 & 0.0057 & 0.043 & 0.0054 & 0.027 & 0.0058 & Hard rock \\
\hline 31 & $2012 / 07 / 10$ & NAUG & 0.045 & 0.0062 & 0.041 & 0.0085 & 0.057 & 0.0084 & Soft soil \\
\hline \multirow[t]{3}{*}{32} & $2012 / 07 / 14$ & GOLA & 0.042 & 0.0051 & 0.033 & 0.0049 & 0.030 & 0.0063 & Soft soil \\
\hline & & JORH & 0.070 & 0.0053 & 0.072 & 0.0040 & 0.045 & 0.0044 & Soft soil \\
\hline & & NAUG & 0.035 & 0.0042 & 0.036 & 0.0058 & 0.047 & 0.0072 & Soft soil \\
\hline
\end{tabular}


Table 3. Comparison of kappa value estimated in present study with those of different regions of world.

\begin{tabular}{cccc}
\hline Sr. no. & Region & Kappa Value & Reference No. \\
\hline 1 & North-eastern Sonora & 0.04 & {$[15]$} \\
2 & California & 0.05 & {$[1]$} \\
3 & France & 0.04 & {$[28]$} \\
4 & Switzerland & 0.015 & {$[34]$} \\
5 & Western Alps & 0.012 & {$[35]$} \\
6 & Central Europe (Germany) & 0.05 & {$[36]$} \\
7 & Guerrero Mexico & 0.045 & {$[11]$} \\
8 & Greece & 0.06 & {$[23]$} \\
9 & Eastern Canada \& Western Canada & 0.04 & {$[37]$} \\
10 & Southern California & 0.06 & This study \\
11 & North-East Himalaya & $0.049(\mathrm{~L}$-comp), & \\
\hline
\end{tabular}

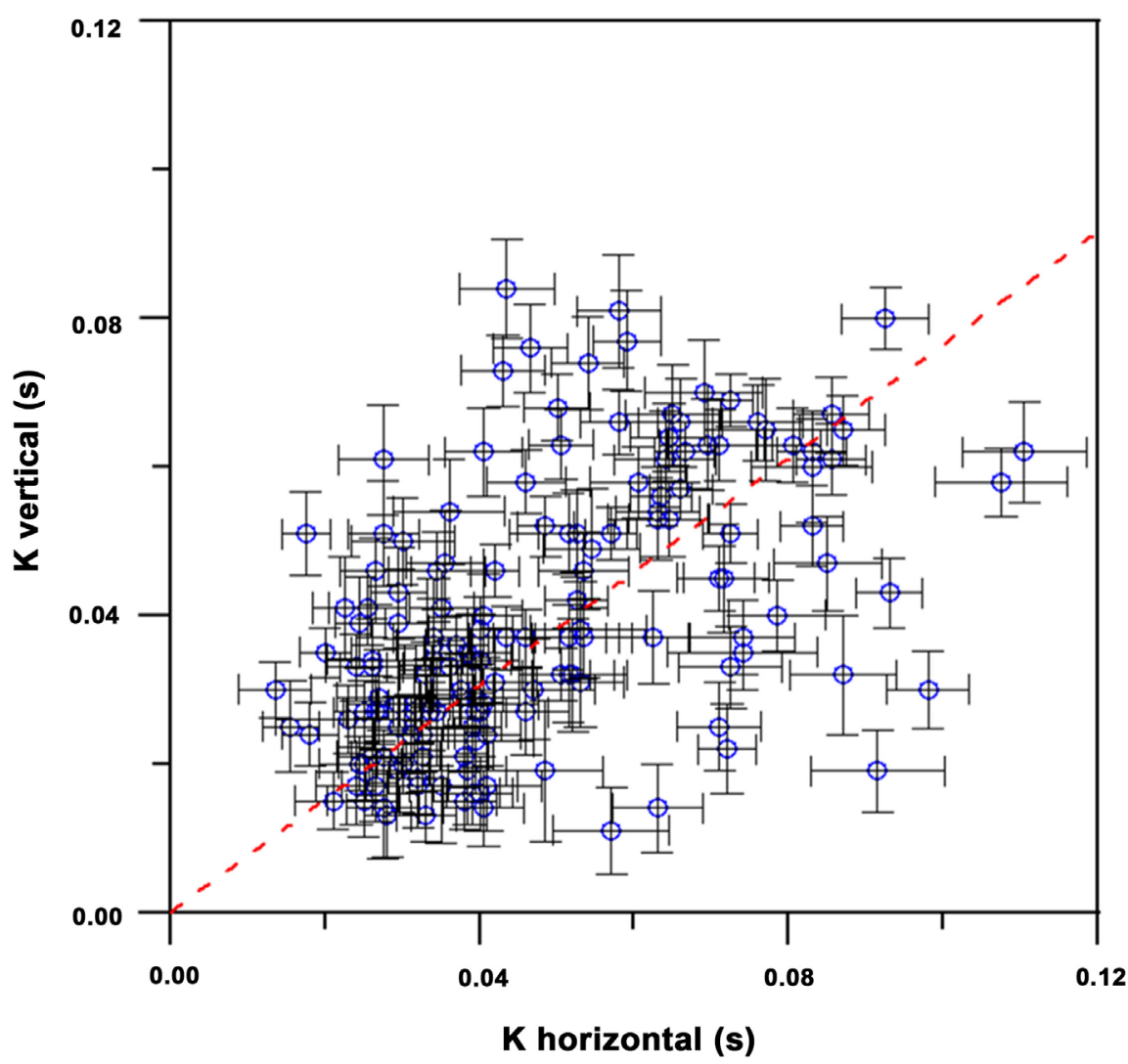

Figure 4. A comparison between the $\kappa$ values obtained from horizontal and vertical components.

[33] has estimated $\kappa_{0}$ values varying in the range $0.032-0.097$ at surface and in the range $0.012-0.078$ in borehole (may be considered as hard rock site) for Taiwan region. [31] has found $\kappa=0.016$ for hard rock site and 0.0201 for soft 


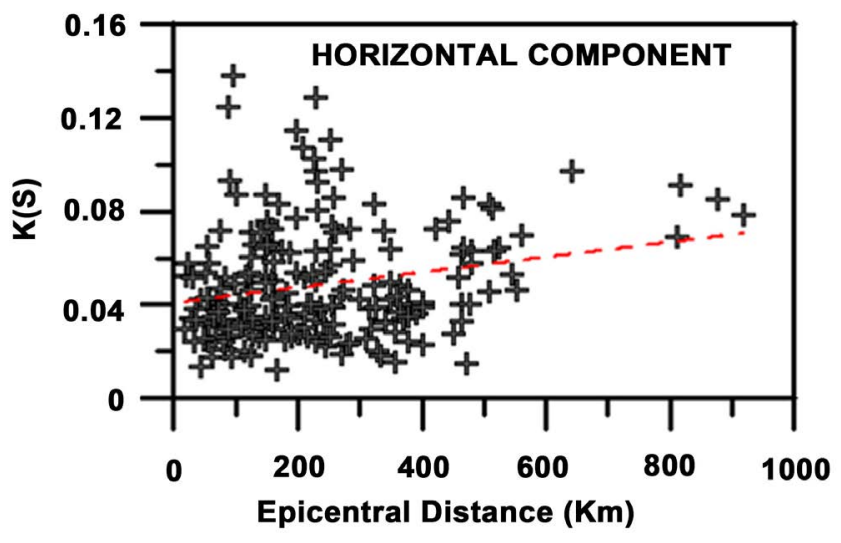

(a)

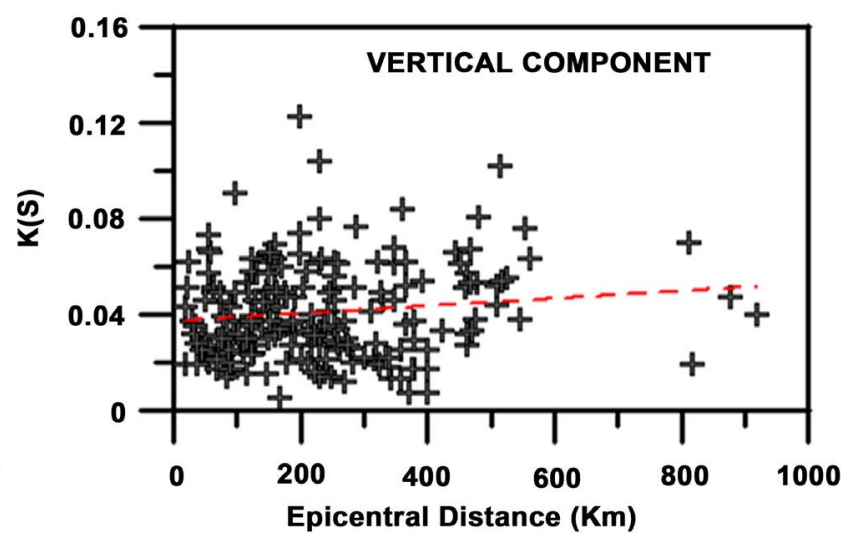

(b)

Figure 5. (a) Dependency of kappa (Horizontal) on Epicentral Distance; (b) Dependency of kappa (Vertical) on Epicentral Distance.

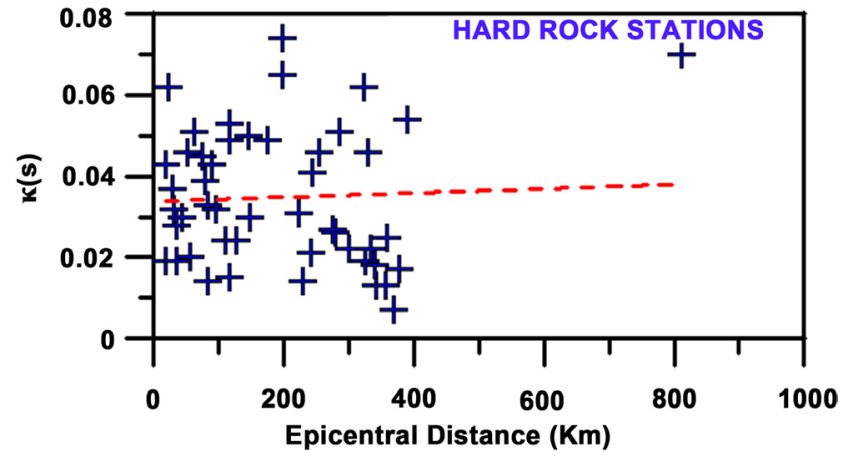

(a)

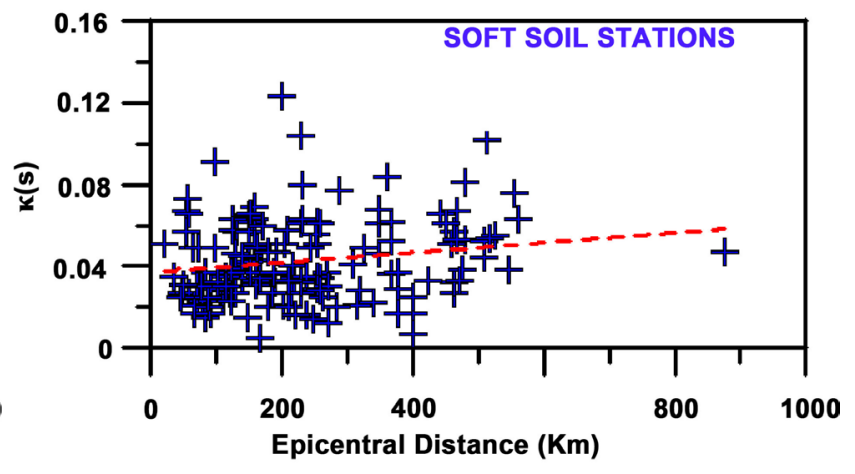

(b)

Figure 6. (a) Distance dependence of $\kappa$ for hard rock sites; (b) Distance dependence of $\kappa$ for soft soil sites. $\kappa=0.034+0.0000158 R$ for hard rock sites and $\kappa=0.037+0.000024 R$ for soft soil sites.
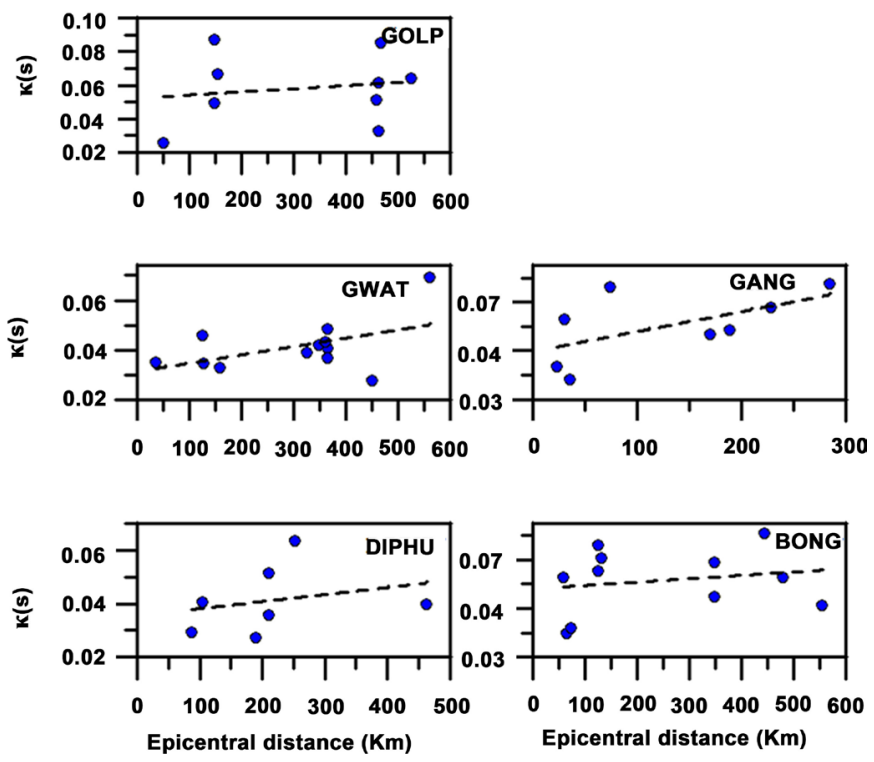

Figure 7. Distance dependence of $\kappa$ for individual station where sufficient numbers of earthquake have been recorded. 

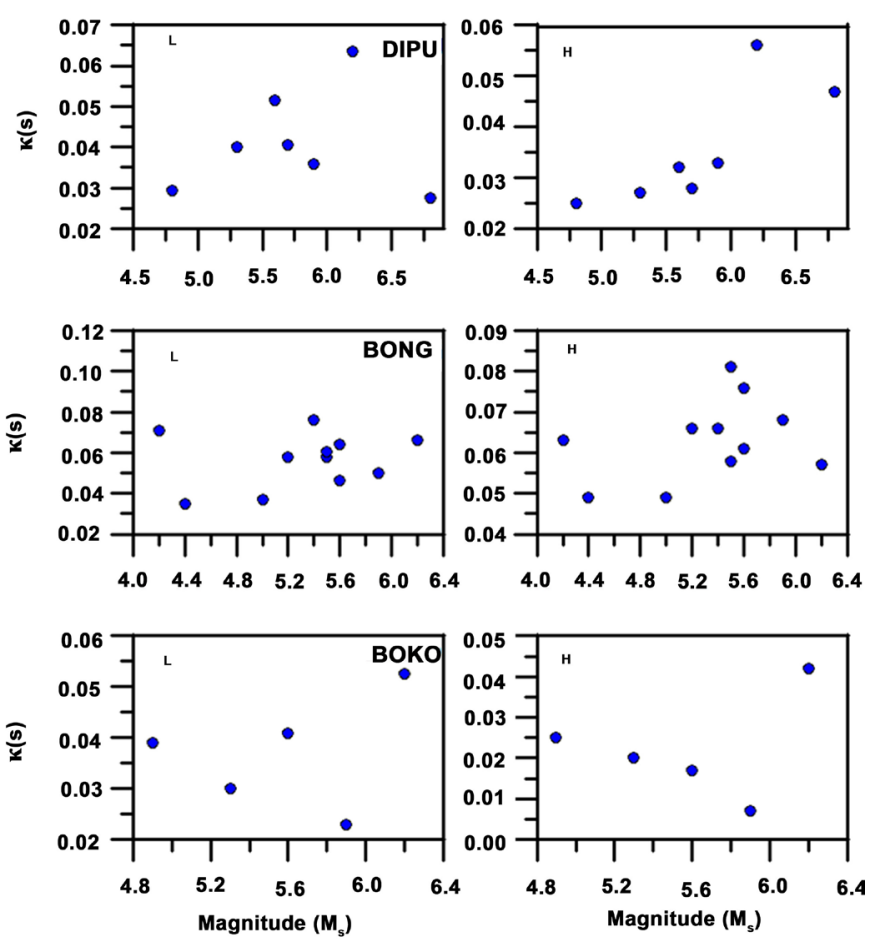

(a)
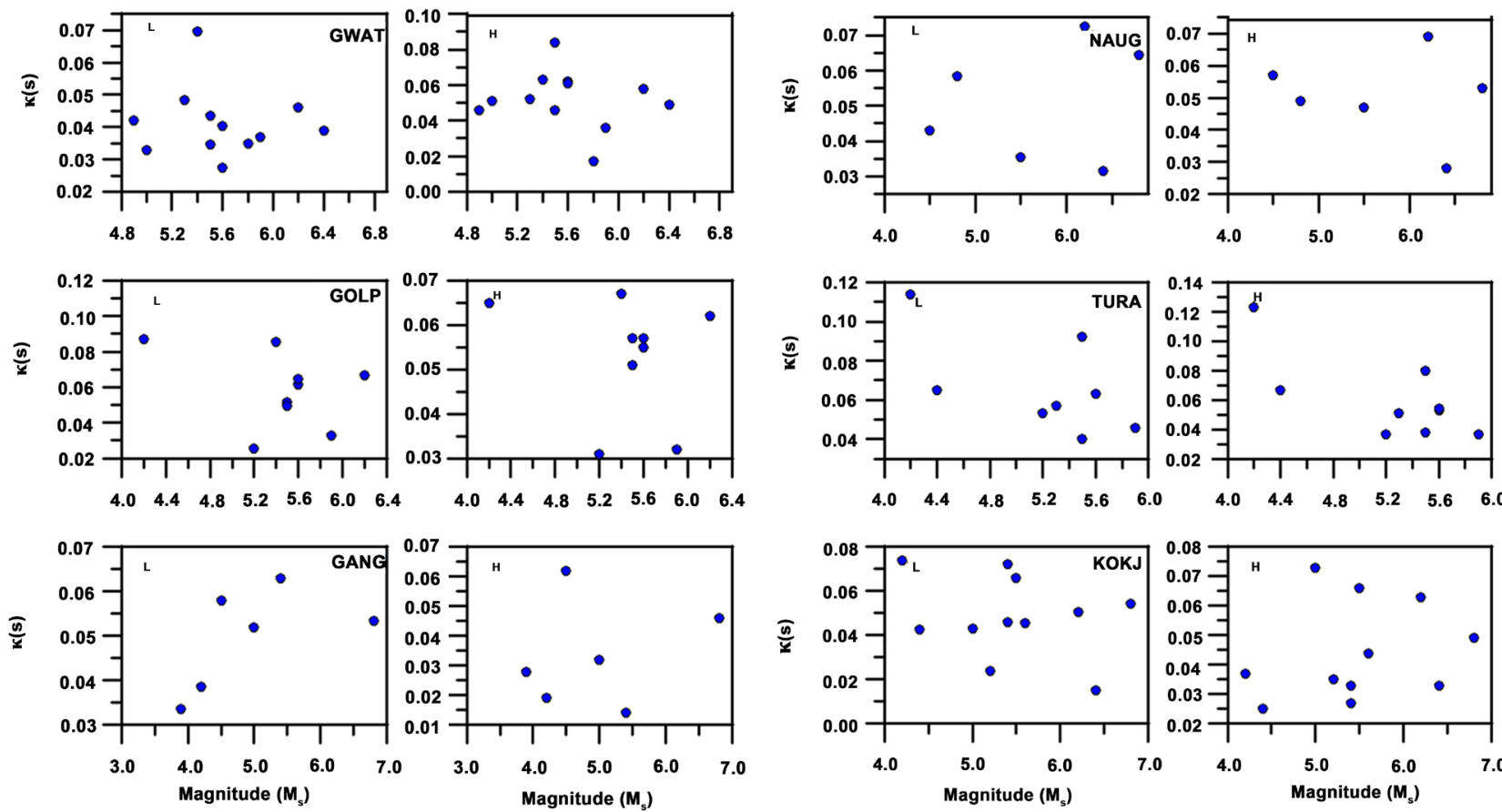

(b)
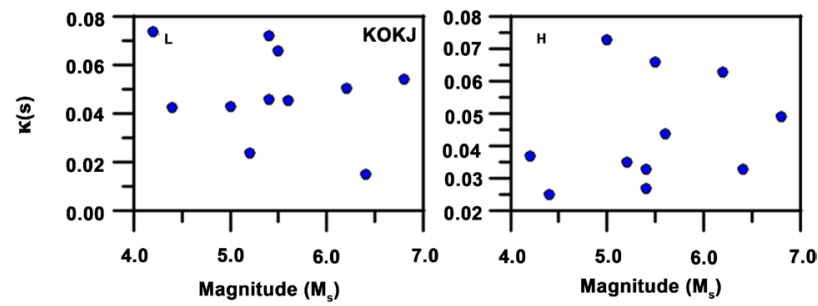

(c)

Figure 8. Magnitude dependency of kappa at some of the stations.

rock site in the Kachchh region of Gujarat, India. Figure 7 shows the plots for the distance dependence of $\kappa$ for individual stations where sufficient number of earthquakes have been recorded. The $\kappa_{0}$ values fall in the range $0.031-0.053$. The change in the $\kappa_{0}$ values for different stations shows the effect of geological 
formations as suggested by [33].

The dependence of kappa values on earthquake size has been examined by plotting the estimated $\kappa$ values with earthquake magnitudes for the stations where sufficient number of earthquake have been recorded. Figure 8(a)-(c) show such plots for some of the stations. We note that there is a scatter and the correlation between " $k$ " and magnitude at most of the stations for the region under study is not significant. This suggests that that " $K$ " is not related to source effect for NE Himalaya region. [31] has reported similar property of $\kappa$ for smaller magnitude earthquakes occurred in Kachchh region of Gujarat, India. The analysis in the present study indicates that kappa for NE region is related with the high frequency attenuation in the top surface layer. One of the scientific discussions about $\kappa$ is whether it is due to source effect or site effect or both. The different studies show different results for different regions of the world. The present study based on the available data found that $\kappa$ is related to site effect in $\mathrm{NE}$ region. This is empirical inference drawn on the basis of recorded waveforms in the region. The same may be validated with more data whenever available.

\section{Conclusions}

The average value of $\kappa$ estimated from the spectral analysis of horizontal components of 598 accelerograms for NE India region has been found to be in the range $0.047-0.049$ and 0.040 for vertical component. The distance dependence of $\kappa$ is not significant. The $\kappa_{0}$ (soft site) $/ \kappa_{0}$ (rock site) ratio is found to be 1.09 . The analysis shows that $\kappa$ is not dependent on the magnitude but dependent on site-condition in the region for the range of magnitudes studies here. The study presents the $\kappa$ model for NE India region which is first study of its kind in the region. The inferences drawn about $\kappa$ in the NE region are based on the data available for the analysis. The same may be validated further as and when more data is available. With more data, the spatial distribution may also be investigated in the region. The other methods reported in the literature may also be applied to estimate $\kappa$ in the region.

The estimated values of $\kappa$ are useful in the studies of Ground Motion Prediction Equations (GMPE) as well as for the simulation of earthquake strong ground motions in the seismically active NE region. Thus this study is important for bearing on the seismic hazard studies of the region.

\section{Acknowledgements}

The authors are thankful to their respective organizations for support. The authors are very grateful to Dr. RBS Yadav for his kind help in this research. The waveform of events has been downloaded from the site http://www.pesmos.in. The authors are thankful to the reviewers and the editor for their extremely constructive comments which helped in improving the manuscript significantly.

\section{References}

[1] Anderson, J.G. and Hough, S.E. (1984) A Model for the Shape of the Fourier Am- 
plitude Spectrum of Acceleration at High Frequencies. Bulletin of the Seismological Society of America, 74, 1969-1993.

[2] Brune, J.N. (1970) Tectonic Stress and Spectra of Seismic Shear Waves for Earthquakes. Journal of Geophysical Research, 75, 4997-5009.

https://doi.org/10.1029/JB075i026p04997

[3] Hanks, T.C. and McGuire, R.K. (1981) The Character of High Frequency Strong Ground Motion. BSSA, 71, 2071-2095.

[4] Hanks, T.C.M. (1982) $f_{\max }$ Bulletin of the Seismological Society of America, 72, 1867-1879.

[5] Papageorgiou, A.S. and Aki, K. (1983) A Specific Barrier Model for the Quantative Description of Inhomogeneous Faulting and Prediction of Strong Motion. I. Description of Model. Bulletin of the Seismological Society of America, 73, 693-722.

[6] Singh, S.K., Apsel, R.J., Fried, J. and Brune, J.N. (1982) Spectral Attenuation of SH Waves along the Imperial Fault. Bulletin of the Seismological Society of America, 72, 2003-2016.

[7] Anderson, J.G. (1986) Implication of Attenuation for Studies of the Earthquake Source. In: Das, S., Boatwright, J. and Scholz, C.H., Eds., Earthquake Source Mechanics, Maurice Ewing Series 6, American Geophysical Union, Washington DC, 311-318. https://doi.org/10.1029/GM037p0311

[8] Anderson, J.G. (1991) A Preliminary Descriptive Model for the Distance Dependence of the Spectral Decay Parameter in Southern California. Bulletin of the Seismological Society of America, 81, 2186-2193.

[9] Tsai, C.-C.P. and Chen, K.-C. (2000) A Model for the High-Cut Process of Strong Motion Acceleration in Terms of Distance, Magnitude and Site Condition: An Examples from the SMART1 Array, Lotung, Taiwan. Bulletin of the Seismological Society of America, 90, 1535-1542. https://doi.org/10.1785/0120000010

[10] Petukhin, A. and Irikura (2000) A Method for the Separation of Source and Site Effects and the Apparent Q Structure from Strong Ground-Motion Data. Geophysical Research Letters, 27, 3429-3432. https://doi.org/10.1029/2000GL011561

[11] Purvance, M.D. and Anderson, J.G. (2003) A Comprehensive Study of the Observed Spectral Decay in Strong-Motion Accelerations Recorded in Guerrero, Mexico. Bulletin of the Seismological Society of America, 93, 600-611. https://doi.org/10.1785/0120020065

[12] Halldorsson, B. and Papageorgiou, A.S. (2005) Calibration of the Specific Barrier Model to Earthquakes of Different Tectonic Regions. BSSA, 95, 1276-1300. https://doi.org/10.1785/0120040157

[13] Boore, D.M. (2003) Simulation of Ground Motion Using the Stochastic Method. Pure and Applied Geophysics, 160, 635-676. https://doi.org/10.1007/PL00012553

[14] Cotton, F., Scherbaum, F., Bommer, J.J. and Bungum, H. (2006) Criteria for Selecting and Adjusting Ground-Motion Models for Specific Target Regions: Application to Central Europe and Rock Sites. Journal of Seismologys, 10, 137-156. https://doi.org/10.1007/s10950-005-9006-7

[15] Fernandez, A.I., Castro, R. and Huerta, C.I. (2010) The Spectral Decay Parameter Kappa in Northeastern Sonora, Mexico. Bulletin of the Seismological Society of America, 100, 196-206. https://doi.org/10.1785/0120090049

[16] Ktenidou, O.-J., Gelis, C. and Bonilla, F. (2013) A Study on the Variability of Kappa in a Borehole, Implications on the Computation Method Used. Bulletin of the Seismological Society of America, 103, 1048-1068. 
https://doi.org/10.1785/0120120093

[17] Campbell, K.W. (2003) Prediction of Strong Ground Motion Using the Hybrid Empirical Method and Its Use in the Development of Ground Motion (Attenuation) Relations in Eastern North America. Bulletin of the Seismological Society of America, 93, 1012-1033. https://doi.org/10.1785/0120020002

[18] Atkinson, G.M. and Boore, D.M. (2006) Earthquake Ground Motions Prediction Equations for Eastern North America. Bulletin of the Seismological Society of America, 96, 2181-2205. https://doi.org/10.1785/0120050245

[19] Dasgupta, S., Pande, P., Ganguly, D., Iqbal, Z., Sanyal, K., Venaktraman, N.V., Dasgupta, S., Roy, A., Das, L.K., Misra, P.S. and Dupta, H. (2000) Seismotectonic Atlas of India and Its Environs. Geological Survey of India, Calcutta, India.

[20] Nandy, D.R. (2001) Geodynamics of North Eastern India and the Adjoining Region. ACB Publications, Kolkata.

[21] Thingbaijam, K.K.S., Nath, S.K., Yadav, A., Raj, A., Walling, M.Y. and Mohanty, W.K. (2008) Recent Seismicity in North-East India and Its Adjoining Region. Journal of Seismology, 12, 107-123. https://doi.org/10.1007/s10950-007-9074-y

[22] Biasi, G.P. and Smith, K.D. (2001) Site Effects for Seismic Monitoring Stations in the Vicinity of Yucca Mountain, Nevada. MOL20011204.0045, A Report Prepared for the US DOE/University and Community College System of Nevada (UCCSN) Cooperative Agreement.

[23] Margaris, B.N. and Boore, D.M. (1998) Determination of $\sigma$ and $\kappa_{0}$ from Response Spectra of Large Earthquake in Greece. Bulletin of the Seismological Society of America, 88, 170-182.

[24] Drouet, S., Cotton, F. and Guéguen, P. (2010) VS30, $\kappa$, Regional Attenuation and Mw from Accelerograms: Application to Magnitude 3-5 French Earthquakes. Geophysical Journal International, 182, 880-898. https://doi.org/10.1111/j.1365-246X.2010.04626.x

[25] Oth, A., Bindi, D., Parolai, S. and Di Giacomo, D. (2011) Spectral Analysis of K-NET and KiK-Net Data in Japan, Part II: On Attenuation Characteristics, Source Spectra, and Site Response of Borehole and Surface Stations. Bulletin of the Seismological Society of America, 101, 667-687. https://doi.org/10.1785/0120100135

[26] Anderson, J.G. and Humphrey Jr., J.R. (1991) A Least-Squares Method for Objective Determination of Earthquake Source Parameters. Seismological Research Letters, 62, 201-209. https://doi.org/10.1785/gssrl.62.3-4.201

[27] Humphrey Jr., J.R. and Anderson, J.G. (1992) Shear Wave Attenuation and Site Response in Guerrero, Mexico. BSSA, 81, 1622-1645.

[28] Douglas, J., Gehl, P., Bonilla, L.F. and Gelis, C., (2010) A $\kappa$ Model for Mainland France. Pure and Applied Geophysics, 167, 1303-1315.

https://doi.org/10.1007/s00024-010-0146-5

[29] Van Houtte, C., Drouet, S. and Cotton, F. (2011) Analysis of the Origins of $\kappa$ (kappa) to Compute Hard Rock to Rock Adjustment Factors for GMPEs. Bulletin of the Seismological Society of America, 101, 2926-2941. https://doi.org/10.1785/0120100345

[30] Motazedian, D. (2006) Region-Specific Key Seismic Parameters for Earthquakes in Northern Iran. Bulletin of the Seismological Society of America, 96, 1383-1395. https://doi.org/10.1785/0120050162

[31] Kumar, S., Kumar, D., Rastogi, B.K. and Singh, A.P. (2018) Kappa ( $\kappa$ ) Model for Kachchh Region of Western India. Geomatics, Natural Hazards and Risk, 9, 
442-455. https://doi.org/10.1080/19475705.2018.1447025

[32] Kilb, D., Biasi, G., Anderson, J., Brnue, J., Peng, Z. and Vernon, F.L. (2012) Spectral Decay Parameter Kappa for Small and Moderate Earthquakes Using Southern California ANZA Seismic Network Data. Bulletin of the Seismological Society of America, 102, 284-300. https://doi.org/10.1785/0120100309

[33] Lai, W., Wei, X., Feng, N.N., Shuping, C., Feng, L. and Qiumei, G. (2016) Research on Seismic Performance of Reinforced Concrete Frame with Unequal Span under Low Cyclic Reversed Loading. The Open Civil Engineering Journal, 10, 373-383. https://doi.org/10.2174/1874149501610010373

[34] Bay, F., Fah, D., Malagnini, L. and Giardini, D. (2003) Spectral Shear-Wave Ground Motion Scaling in Switzerland. Bulletin of the Seismological Society of America, 93, 414-429. https://doi.org/10.1785/0120010232

[35] Morasca, P., Malagnini, L., Akincl, A., Spallaroosa, D. and Hermann, R.B. (2006) Ground Motion Scaling in Western Alps. Journal of Seismology, 10, 315-333. https://doi.org/10.1007/s10950-006-9019-x

[36] Malagnini, L., Herrmann, R.B. and Koch, K. (2000) Regional Ground Motion Scaling in Central Europe. Bulletin of the Seismological Society of America, 90, 1052-1061. https://doi.org/10.1785/0119990151

[37] Atkinson, G.M. (1996) The High Frequency Shape of the Source Spectrum for ZEarthquakes in Eastern and Western Canada. Bulletin of the Seismological Society of America, 86, 106-112. 